\title{
Fascination of Down Scaling - Alice the Sugar Cube Robot
}

\author{
G. Caprari, T. Estier, R. Siegwart \\ Institute of Robotics System \\ Swiss Federal Institute of Technology Lausanne (EPFL), Switzerland \\ E-mail: Gilles.Caprari@epfl.ch \\ http://dmtwww.epfl.ch/isr/asl
}

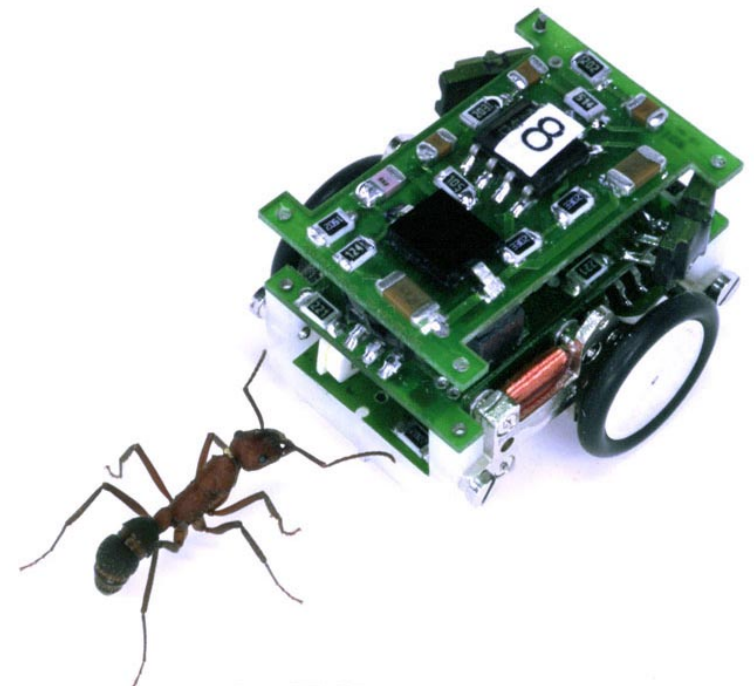

Figure 1: The size of Alice is similar to an ant.

\begin{abstract}
:
Design of mobile micro-robot (MMR) is still a challenge due to the restricted availability of basic components. However, the number of highly integrated microelectronic and micromechanical components is growing fast. Nevertheless, its integration to a micro-system requires a good knowledge of all the interactions between sensor, actuator, computation and energy source. Often compromises between performance and power consumption have to be found.

This paper gives the basic considerations for building mobile micro-robots. The major scaling effects are presented and their impact on micro-system design is discussed. The mobile micro-robot Alice (fig. 1), having the size of a sugar cube, is presented and discussed in the context of scaling laws. It has an autonomy of around 10 hours and is able to navigate based on simple behaviors like obstacle avoidance or wall following.
\end{abstract}

\section{Introduction}

With the progress in micro- and nano-technologies (MNT), a large number of sensors and actuators operating in submillimetric range appeared. Subsequently, various research groups started recently to develop micro-robotic systems for a wide range of application: precision tooling, endoscopic surgery, biological cells manipulation, AFM microscopy, etc. However, these devices are not really autonomous, neither concerning energy supply nor intelligence. But autonomy is a major issue for a lot of innovative application of microrobots where teleoperation is not possible or not desirable. Typical examples might be found in medicine, where micro- robots could deliver a medicament exactly were needed or in inspection and exploration, where a much larger surface can be covered when using a large quantity of micro-robots [Garcia97]. To advance towards such very challenging applications, it is of high importance to identify the key parameters that limit downscaling [Nicoud95]. Mechanical miniaturization has already been investigated several decades ago [Burckhardt72]. As most of the envisioned micro-robots are still in the domain of classical physics, the classical theory is still applicable to establish the basic scaling properties of the domains in question. Scaling laws express the dependence to a scale effect for physical parameters and are usually expressed in function of a reference length $\mathrm{L}$. Two procedures are possible: applying an isotropic modification of the dimensions or holding some parameters invariant with respect to the scale and study their influence on the other parameters. The aim of this article is to discuss and analyze the scaling laws and to verify some aspects on an existing mobile micro-robot.

This paper is organized in two major sections. The first section highlights different scaling aspects for MMR, trying to be as general as possible. In the second section the microrobot Alice is presented and discussed as a case study.

Within our current research activities we concentrate on robots sizing between a couple of millimeter up to a couple of centimeters. All the text long we will use $L$ as the characteristic length of reference.

\section{Laws of Scaling}

Within this section we will discuss the most important scaling effects related to mobile micro-robots. The discussion is not exhaustive, however it should give a basic understanding of the scaling effects and its consequences on the design of mobile micro-robots.

\subsection{Mechanics}

The first where the similarity method can be applied is the mechanics of the robot. Mechanical properties are well understood and scaling effects have been investigated and verified for several decades [Drexler92].

The dimensions related to the volume (mass, inertia) are proportional to $L^{3}$, whereas dimensions related to the area (cross section) scale down only with the exponent 2 . Moreover the structural stiffness and the stress related to the mass scale linearly with $L$. This is a great advantage for smaller systems which are intrinsically more robust against destruction forces related to their own mass. In the design we 
can thus choose thinner and less bulky structures or weaker materials still conserving a good rigidity. An evident example is found in nature if one compares the cross section of the leg of an elephant with that of an ant. Additionally the structural eigenfrequencies increase linearly with $1 / L$, thus we can expect less interference with structural resonances. Surface friction depends on the normal force, which is proportional to the mass $\left(L^{3}\right)$. But the energy $E_{\mu}$ lost by friction scales down more importantly assuming relative displacements proportional to $L$. This is true for small motors or gears, where one can use slider bearing instead roller bearings. However, it holds only if we assume to keep the rotational speed constant, which is not the case for many components.

\begin{tabular}{|c|c|c|}
\hline Unit & Scaling & Comments \\
\hline $\begin{array}{l}\text { Volume, } \\
\text { Mass: }\end{array}$ & $Q \sim L^{3} ; m \sim L^{3}$ & \\
\hline $\begin{array}{l}\text { Mass related } \\
\text { force: }\end{array}$ & $F \sim m \sim L^{3}$ & $\begin{array}{l}\text { acceleration, } \\
\text { gravity, } \\
\text { impact forces }\end{array}$ \\
\hline $\begin{array}{l}\text { Friction } \\
\text { forces: }\end{array}$ & $F_{\mu}=\mu \cdot F \sim L^{3}$ & $\begin{array}{l}\text { e.g. contact } \\
\text { between wheel } \\
\text { and surface }\end{array}$ \\
\hline $\begin{array}{l}\text { Energy } \\
\text { losses due } \\
\text { to friction: }\end{array}$ & $E_{\mu}=L \cdot F_{\mu} \sim L^{4}$ & $\begin{array}{l}\text { assuming that } \\
\text { displacement } \\
\text { scales with } L\end{array}$ \\
\hline $\begin{array}{l}\text { Potential } \\
\text { energy: }\end{array}$ & $E_{p} \sim h \cdot m \sim L \cdot L^{3}=L^{4}$ & \\
\hline $\begin{array}{l}\text { Wind, lift, } \\
\text { drag forces: }\end{array}$ & $F_{w} \sim L^{2}$ & \\
\hline Stiffness & $\begin{array}{l}c \sim L \\
c \sim \frac{b \cdot h^{3}}{l^{3}} \sim L\end{array}$ & of a beam \\
\hline \multirow[t]{2}{*}{ Stress: } & $\sigma \sim \frac{F}{L^{2}}$ & $\begin{array}{l}\text { beam exposed } \\
\text { to an external } \\
\text { force } F\end{array}$ \\
\hline & $\sigma_{\max } \sim \frac{F \cdot l}{b \cdot h^{2}} \sim \frac{L^{3} \cdot l}{b \cdot h^{2}} \sim L$ & $\begin{array}{l}\text { beam expose } \\
\text { to its own } \\
\text { mass }\end{array}$ \\
\hline $\begin{array}{l}\text { Structural } \\
\text { eigen- } \\
\text { frequencies: }\end{array}$ & $\begin{array}{l}f_{0} \sim \frac{1}{L} \\
f_{0}=\sqrt{\frac{c}{m}} \sim \sqrt{\frac{L}{L^{3}}} \sim \frac{1}{L}\end{array}$ & elastic beam \\
\hline
\end{tabular}

Table 1: scaling of some mechanic quantities.

Air drag and lift depend on the area and thus are proportional to $L^{2}$. This can be an advantage but also a disadvantage in some cases. Due to this effect, a flying robot might fly much slower, thus simplifying the control. However, secondary fluid effect might play a dominant roll for small systems. A falling miniature robot has much better chance to survive because of the increasing ratio between air drag and mass. This effect is additionally supported by the mechanical properties mentioned above. On the other hand a small land robot is much more exposed to wind and friction with the terrain could be insufficient for staying stationary (friction $\sim L^{3}$, drag $\sim L^{2}$ ). These air drag effects become also very evident in nature. A small animal, e.g. an ant, can easily survive a fall from a multi floor building, whereas an elephant will be seriously hurt when falling from around 1 meter.

\subsection{Scaling effects on mobility}

The size of a mobile robot has strong implications on the mobility in a given environment. Wilcox [Wilcox97] introduced the "mean free path" as the average distance a robot can move before it encounters a non-traversable obstacle. This distance depends on the size of the robot, the maximal surmountable obstacle height and the terrain morphology (rock distribution and size). For a given terrain there is a range of optimal dimensions. However, it should be noticed that small robots can surmount higher obstacles in relation to its size. This is due to the fact that the required energy to get on an obstacle of its own height is proportional to $L^{4}\left(\mathrm{E}_{\mathrm{pot}} \sim \mathrm{h} \cdot \mathrm{m} \sim \mathrm{L} \cdot \mathrm{L}^{3}\right)$ and thus decrease faster then the mass (volume). Evident examples from the biology are again small insects against big animals. Insects climb relatively high obstacles in their daily live. Some of them e.g. fleas or grasshoppers even prefer jumping. They jump over $20 \mathrm{~cm}$, which is in the same order of magnitude as a jump of humans.

Another aspect of mobility is the speed of movement. Observations in nature and of autonomous mobile robots show that the speed scales approximately linear with L. There are of course large variations in velocity between different species/robots of the same mass, but in average the linear scaling law holds. As we will see later, this is related to power, energy, control and also sensors of MMR.

\subsection{Actuators}

Actuators are one of the major problems in designing miniature robots. The main reason is the lack of commercially available micromotors and the low performance of the existing ones. For a good overview on electrostatic and electromagnetic actuators please refer to [Dario92] and [Fearing98].

Most actuators such as combustion engines, pneumatic, electromagnetic, electrostatic, ultrasonic, shape memory alloy, piezoelectric or biological muscle used for large systems might also be useable in micro-robots. However, some of them might be very difficult to build in small size and thus are useless for our investigation. Others are very 
interesting and promising but not yet well developed and present important drawbacks (low speed, high voltage, low forces, ...). One of the most promising and interesting solutions in long term might be artificial muscles that show excellent scalability in nature. However, they are still far from real applications.

One of the actuators that are already available in small scale are electromagnetic motors. For scaling of electromagnetic motors we can find for the torque $M$ [Jufer95]:

- $\quad M \sim L^{5}$, assumption constant efficiency

- $\quad M \sim L^{3.5}$, assuming similar motor temperatures.

In reality the scaling might lay somewhere in-between the two assumption above. Although this scaling effect of the motor torque is somewhat unfavorable, electrical motors still represent one of the most interesting solutions because of their availability (see table 2) and ease of control.

\begin{tabular}{|l|r|r|r|r|r|}
\hline \multicolumn{1}{|c|}{$\begin{array}{c}\text { Motor } \\
\text { Type }\end{array}$} & $\begin{array}{r}\text { torque } \\
{[\mathrm{mNm}]}\end{array}$ & $\begin{array}{r}\text { power } \\
{[\mathrm{mW}]}\end{array}$ & $\begin{array}{r}\text { speed } \\
{[\mathrm{rpm}]}\end{array}$ & $\begin{array}{c}\text { volume } \\
{\left[\mathrm{mm}^{3}\right]}\end{array}$ & $\begin{array}{c}\text { eff. } \\
{[\%]}\end{array}$ \\
\hline $\begin{array}{l}\text { Smoovy } 3 \\
\text { gear 1:125 }\end{array}$ & 2.0 & 12 & 120 & 110 & 6 \\
\hline $\begin{array}{l}\text { Minimotor } \\
1.9 \text { mm } \\
\text { gear 1:47 }\end{array}$ & 0.15 & 3 & 425 & 27 & 8.25 \\
\hline $\begin{array}{l}\text { Wobble } \\
\text { from SSSA }\end{array}$ & 0.35 & 2.6 & 185 & 195 & 4.7 \\
\hline $\begin{array}{l}\text { ETA lavet } \\
\text { by Swatch }\end{array}$ & 0.35 & 1.3 & 60 & 210 & 6.3 \\
\hline
\end{tabular}

Table 2: Examples of small motors (tested in a limited temperature range).

For an autonomous robot a motor with a good energetic efficiency should be preferred, but as torque scale with $\mathrm{L}^{5}$, the mechanical power become very fast smaller for decreasing $L$. In practice the volume of the coil become predominant over the magnet, increasing significantly the overall size. On the contrary, the motor designers are mainly interested in a good power to mass ratio, and normally prefer a much lower efficiency and a higher working temperature. This is allowed because the surface to volume ratio $\left(L^{2} / L^{3}=\right.$ $L^{-1}$ ) responsible for heat dissipation is scaling favorably.

Assuming constant surface speed on the rotor, the rotational speed $\Omega$ of a motor scales with $1 / L$. Thus small motors require gearboxes with high reduction rates which additionally reduce the efficiency.

\subsection{Energy source}

Mobile micro-robots (MMR) require an on-board energy source or the capability to generate energy from an external source. The most obvious energy sources are batteries, accumulators, supercaps, springs, fuel or solar cells. The scaling properties and power density of the different energy sources are presented in table 3.
Among the electrical energy storages, batteries have the highest power density and an excellent availability in many different sizes. Accumulators and supercaps have lower energy density but are rechargeable, thus also compatible with power generators (e.g. solar cells). The energy density of supercaps is very limited, but, compared to accumulators, they allow much higher currents for charging and discharging.

Fuel has a very high energy density, but it might be a great challenge to build small size combustion engines and generators. The technology is not available today.

Finally, solar panels become interesting in small size because of their advantageous downscaling effect. However, in most cases they have to be combined with accumulators or supercaps.

\begin{tabular}{|l|c|r|r|}
\hline $\begin{array}{c}\text { Energy } \\
\text { Source }\end{array}$ & scaling & \multicolumn{1}{c|}{$\begin{array}{c}\text { energy } \\
{\left[\mathrm{Wh} / \mathrm{I}^{3}\right]}\end{array}$} & \multicolumn{1}{c|}{$\begin{array}{c}\text { energy } \\
{[\mathrm{Wh} / \mathrm{Kg}]}\end{array}$} \\
\hline Battery & $L^{3}$ & $230-1300$ & $51-408$ \\
\hline Accu & $L^{3}$ & $40-210$ & $11-61$ \\
\hline Supercap & $\sim L^{4}$ & $1.8\left(@ 9 \mathrm{~cm}^{3}\right)$ & $1.6\left(@ 9 \mathrm{~cm}^{3}\right)$ \\
\hline Spring & $L^{3}$ & 0.07 & 13000 \\
\hline Fuel & $L^{3}$ & 13000 & \\
\hline Solar panel & $L^{2}$ & $0-100 \mathrm{~W} / \mathrm{m}^{2}$ & \\
\hline
\end{tabular}

Table 3: Energy sources.

The scaling properties given in table 3 are derived form real examples and some simplified models not considering the housing. Consequently the given values might be somewhat optimistic. However, taking into account current technology and availability, the most promising power sources for MMR are still batteries or the combination of solar cells and accumulators.

\subsection{Sensors}

As we are interested in autonomous mobile robots, sensors for environment perception are of high importance (table 4). In relation with scaling, the power consumption of sensors might become the major issue. Generally, we can distinguish between passive and active sensors. Passive sensors do not irradiate energy in to the environment (e.g. camera, microphone) whereas active sensors send out some sort of signals that support the measurement.

In consequence, the power consumption of passive sensors is dominated by the signal conversion and processing, which is barely changing with the robot size. In contrast, active distance sensors like sonar, IR proximity sensors, triangulation (PSD), light stripe, laser range finder, magnetic or radar emit energy and use the reflected beams dispersed by the object to measure. We might assume that the required measurement distance is proportional to the characteristic length $L$, which makes sense if the robot speed scales linearly. Under this assumption, the emission power depends on $L^{2}$ (surface of the sphere where the reflection is dispersed) 
multiplied by an exponential factor function of $L\left(e^{k L}\right.$ representing the energy dissipation). However, even if $L^{2} \cdot e^{k L}$ is favorable for small size, active sensors might have power consumptions that are not feasible for MMR. Thus passive sensors or very simple active sensors are the right choice for small systems.

\begin{tabular}{|c|c|c|c|}
\hline sensor & principle & output & comment \\
\hline bumpers & contact & on-off & easy \\
\hline compass & magnetic & angle & feasible \\
\hline inclinometer & inertia & angle & feasible \\
\hline barometer & pressure & bar & feasible \\
\hline temperature & heat & ${ }^{\circ} \mathrm{C}$ & easy \\
\hline microphone & sound & $\mathrm{Hz}$ & feasible \\
\hline photodiode & light & LUX & feasible \\
\hline camera & $\begin{array}{l}\text { light, } \\
\text { position \& } \\
\text { color }\end{array}$ & $\begin{array}{l}\text { value per } \\
\text { pixel }\end{array}$ & $\begin{array}{c}\text { not easy } \\
\text { and much } \\
\text { information }\end{array}$ \\
\hline sonar & $\begin{array}{c}\text { time of } \\
\text { flight }\end{array}$ & proximity & $\begin{array}{l}\text { not for short } \\
\text { range }\end{array}$ \\
\hline infrared & $\begin{array}{l}\text { reflected } \\
\text { light }\end{array}$ & proximity & feasible \\
\hline PSD & $\begin{array}{l}\text { reflected } \\
\text { angle }\end{array}$ & distance & $\begin{array}{c}\text { to be } \\
\text { integrated }\end{array}$ \\
\hline laser ranging & $\begin{array}{l}\text { time of } \\
\text { flight }\end{array}$ & distance & difficult \\
\hline
\end{tabular}

Table 4: Possible sensors for MMR.

\subsection{Control, Processing}

The controller of a robot has to process information and generate adequate actions. This task might not change much with the size of the robot. However, we might argue, that smaller system have a more restricted, thus less complex environment to deal with. This might allow a group of small robots to fulfill a task with lower computational power per robot. Additionally, small robots are moving slower, thus requiring less demanding sampling times for reaction. However, these effects will in most cases not compensate for the important reduction of calculation power with size.

As we have seen above, the available energy of a MMR scales with $L^{3}$. The required power $P_{C P U}$ of a microprocessor is related to the number of transistors $n$, the clock frequency $f$ and the power supply $V_{d d}$.

$$
P_{C P U} \sim n \cdot f \cdot V_{d d}^{2}
$$

If we assume $f \sim$ velocity $\sim L$, the power consumption scales linear with $L$. However, because the available power is scaling with $L^{3}$, we still have to admit a reduction in calculation power by $L^{2}$, thus drastically limiting control capacity. It is therefore a must and not a choice to further reduce the calculation power by using 8-bit instead of 16 or 32-bit microcontrollers.

In consequence, we have to admit that the intelligence of MMR will be limited. Nevertheless, in connection with an external supervisor (computer, human) or an adequate collective approach, small robot might still be able to fulfill complex tasks.

\subsection{Communication}

Communication in MMR takes place between different units or between the robot and the supervisor or user. The communication can be uni- or bi-directional, involving a receiver, a transmitter or both on the robot.

The power consumption and the dimension of communication devices often depend less on the communication distance but more on the precision, the conversion technique and the communication speed.

Receivers have relatively low power consumption but as they are almost always operating, it becomes an important power drain for MMRs.

The transmitters irradiate power like an active sensor. Thus the power consumption depends on $L^{2} \cdot e^{k L}$, where $L$ is the communication distance that we assume again proportional to the size of the MMR.

Communication can be established through infrared, visual signaling, sound or radio. For short range, infrared becomes interesting because of the favorable $L^{2} \cdot e^{k L}$ scaling and it is much simpler than radio. Moreover, clever combinations of different concepts for the sender and the receiver might reduce power consumption. For example the robot could receive infrared signals and answer with a particular movement or it could use its IR distance sensor also for communication. In any case, communication is quite power consuming for a MMR and thus should be reduced to a minimum. This favors solutions where the robot operates autonomously using its on-board capabilities only.

\section{The mobile micro-robot Alice}

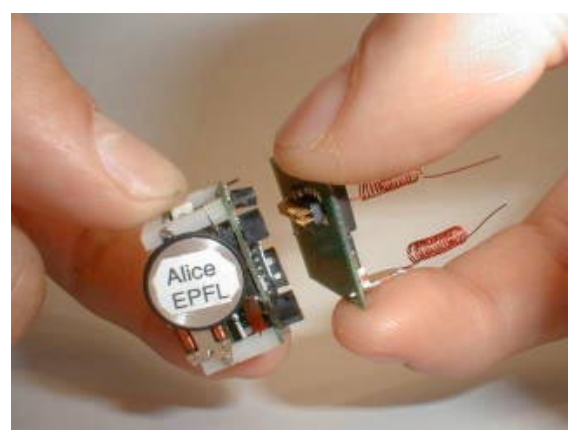

Figure 2: The mobile micro-robot Alice with its motor and sensor module assembled and the chassis (left) and a radio module (right).

The mobile micro-robot Alice is presented here as a case study to demonstrate and verify the scaling law discussed 
above. It is an excellent example of a miniaturized and highly integrated mechatronic product.

Alice is one of the smallest intelligent mobile robots of the world (table 5-7). The remarkable long power autonomy of about 10 hours makes it very particular and unique in its class. Simplicity, modularity, hardware and software flexibility, robustness and affordable price are further advantages. Maybe these features and the accumulated experience were decisive for the victory at the Micro Maze Contest of the last two years in Nagoya, Japan.

In agreement with the conclusions drawn from the section on mechanical scaling, Alice can fall down from 1 meter without serious damage and can fall down twice its own height without any problem $(\sim 5 \mathrm{~cm})$.

\begin{tabular}{|l|l|}
\hline Dimensions & $21 \times 21 \times 12 \mathrm{~mm}$ \\
\hline Weight & $5 \mathrm{~g}$. \\
\hline Velocity & $40 \mathrm{~mm} / \mathrm{s}$ \\
\hline Power consumption & $4 \mathrm{~mW}-10 \mathrm{~mW}$ \\
\hline System autonomy & up to 10 hours \\
\hline Infrared remote communication & $6 \mathrm{~m}, 500 \mathrm{bps}$ \\
\hline Infrared local communication & $4 \mathrm{~cm}, 500 \mathrm{bps}$ \\
\hline Radio communication & $10 \mathrm{~m}, 1000 \mathrm{bps}$ \\
\hline
\end{tabular}

Table 5: General characteristic of Alice.

In contrast to big robots, the supporting structure is made of plastic and printed circuit board. The material cost is only of about $\$ 50$, whereas the assembling time for the prototypes is still approximately 3 hours. An automated assembly is feasible for high quantity production.

Table 5 gives the main characteristics of the robot. Alice reaches a maximum velocity of $40 \mathrm{~mm} / \mathrm{s}$ (twice the length) that demonstrates the scaling linear with $L$. Bigger robots of about 1 cubic meter run about at $1-3 \mathrm{~m} / \mathrm{s}$.

The power consumption of the motors of $3 \mathrm{~mW}$ is about $30 \%$ of the total power consumption and thus still very important part (table 7). Moreover it should be noticed that the motors, being probably the most critical component, mainly give the size of Alice.

\begin{tabular}{|l|l|}
\hline Mechanical structure & plastic frame and PCB \\
\hline Motors & 2 Swatch motors \\
\hline Motion & 2 wheels on the minute axis \\
\hline Energy source & $\begin{array}{l}\text { 3 button batteries }(1.5 \mathrm{~V}, 23 \mathrm{mAh}) \\
\text { + voltage regulator }\end{array}$ \\
\hline Sensors & 4 infrared proximity sensors \\
\hline CPU & PIC16F84 @ 4 MHz \\
\hline Communication 1 & $\begin{array}{l}\text { Local with the same 4 proximity } \\
\text { sensors }\end{array}$ \\
\hline Communication 2 & $\begin{array}{l}\text { One way IR with dedicated circuit } \\
\text { (1 diode + 2 OpAmps) }\end{array}$ \\
\hline Communication 3 & One way IR with RC5 standard \\
\hline Communication 4 & Both ways radio. On-off keying \\
\hline
\end{tabular}

Table 6: principal parts of Alice.
As argued in chapter 2.4, also for Alice, chemical batteries are the best power source. Because of the limited power of these button batteries, a voltage regulator was used to ensure a stable supply voltage.

Active proximity sensors are used for environment perception. They are very simple to use and have a limited range of 2 to $3 \mathrm{~cm}$ (similar to $L$ ). To minimize power consumption, measurements are taken only every $50 \mathrm{~ms}$. This is still enough for reliable obstacle avoidance at the robot speed. In this particular case we see again how energy, sensors, control and mobility are closely connected and a compromise was found.

\begin{tabular}{|l|c|c|}
\hline \multicolumn{1}{|c|}{ unit } & average $[\mathrm{mW}]$ & peak $[\mathrm{mW}]$ \\
\hline motors $(1 \mathrm{x})$ & 1.5 & 2.5 \\
\hline CPU & 3 & \\
\hline sensors & 0.5 & 18 \\
\hline infrared rx & 0.6 & 1 \\
\hline local communication & & 18 \\
\hline radio rx & 3 & 3 \\
\hline radio tx & & 30 \\
\hline The robot & $\sim 10$ & $\sim 45$ \\
\hline
\end{tabular}

Table 7: power consumption of each subsystem.

Another main power drain is the microprocessor, also using $30 \%$ of the total power. The program is written in Assembler, allowing to optimize the code reduce memory space to a minimum. Behaviors like obstacle avoidance or wall following are implemented on the microcontroller.

Finally different ways of communications are available:

- Unidirectional IR communication for teleoperation based on the behaviors like obstacle avoidance or wall following.

- Bi-directional IR communication with distance sensors for robot to robot short range communication.

- Bi-directional radio communication used for communication with an external supervisor. This allows for automatic map building in simple labyrinths.

When possible it is used the less power consuming one way infrared communication and an external camera [Siegwart98]. In this case the user send a signal (command) to the robot and the feedback is given by the behavioral reaction of the robot.

The robot Alice is already used in various research and educational projects:

- localization and map building,

- local and global planning methods with hybrid (metrictopological) environment models,

- control strategies for semi-autonomous operation via Internet or matlab,

- robot soccer tournaments,

- research platform for biologists and

- ludic applications.

Furthermore, Alice is an inspiration for other mobile robots and autonomous systems in general. For the first prototype of 
LAMAlice (fig. 3) essentially we connected 2 Alices, we put bigger flexible blade wheels and used the same IR receiver module to perform the first drive tests [Freese99]. We were able to build very fast an off-road prototype using the same concept and very similar hardware.

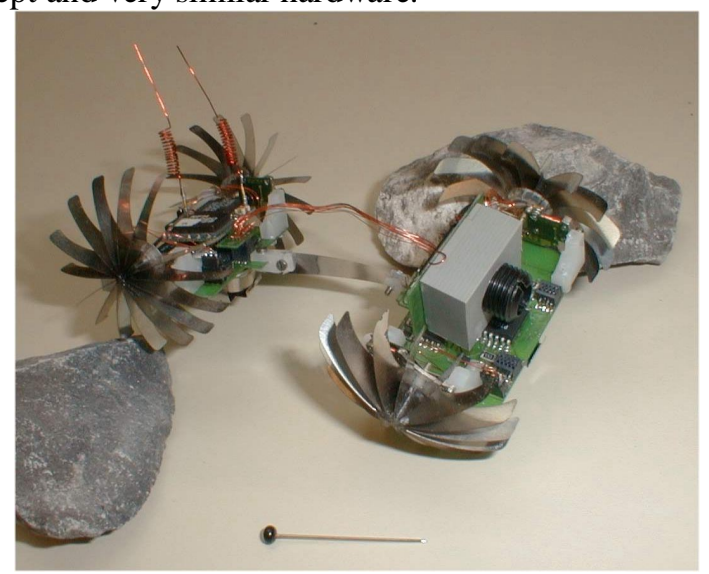

Figure 3: LAMAlice: The off-road sister of Alice.

\section{Conclusion}

In this paper we presented and discussed the advantages and the limitations of a mini mobile robot (MMR). More in detail, we listed and looked in the composing parts for a better understanding, before seeing the interconnections in a real built robot. More critical than for bigger robot, the design of a MMR has to carefully consider these dependencies for a good choice of the single parts composing the whole. The lack of high performance motors, sensors and power sources will force to research in the specific fields, attacking the problems once at the time. The conception of a MMR can only take advantage of the necessary future improvements of the different subsystems. At that moment a further effort will have to be done in order to put all together having an overview of the state of the art.

\section{References}

[Garcia97] C.Garcia Marirrodriga, M.Van Winnendael, P.Putz, "Micro-Rovers for Scientific Applications in Mars or Moon Missions", ESTEC, 1997.
[Burckhardt72] C.W. Burckhardt, "Les lois de similitude en microtechnique", Journées de microtechnique, pp 6-19, 1972.

[Jufer95] M. Jufer, "Electromécanique”, Traité d'électricité, Vol. IX, Presses Polytechniques et Universitaires Romandes, Lausanne, 3ème édition, 1995.

[Drexler92] E. Drexler, "Nanosystems: Molecular Machinery, Manufacturing, and computation", John Wiley \& Sons, Inc, 1992.

[Wilcox97] B. Wilcox, A. Nasif and R. Welch, "Implications of Martian Rock Distributions on Rover Scaling", Planetary Society International Conference on Mobile Planetary Robots and Rover Roundup, Santa Monica CA, January 29 - February 1, 1997.

[Dario92] P. Dario et al., "Microactuators for microrobots: a critical survey", Journal of Micromechanics and Microengineering, vol 2, no 3, p.141-57, sept. 1992.

[Fearing98] R.S. Fearing, "Powering 3 Dimentional Microrobots: Power density Limitations", Tutorial on Micro Mechatronics and Micro Robotics, ICRA 1998.

[Nicoud95] J-D. Nicoud, "Microengineering: when is small too small? Nanoengineering: when is large too large?", Int. Symposium on Micro Machine and Human Science, pp. 1-6, 1995.

[Caprari98] G. Caprari, P. Balmer, R. Piguet, R. Siegwart, "The Autonomous Micro Robot ALICE: A platform for Scientific and Commercial Applications", Int. Symposium on Micromechatronics and Human Science, pp 231-5, 1998.

[Siegwart98] R. Siegwart, C. Wannaz, P. Garcia, R. Blank, "Guiding Mobile Robots through the Web", Workshop Proc. of IEEE/RSJ International Conference on Intelligent Robots and Systems, Victoria, Canada, October 12-16, 1998

[Freese99] M. Freese, M. Kaelin, J-M. Lehky, G. Caprari, T. Estier, R. Siegwart, "LAMAlice : A nanorover for planetary exploration", Int. Symp. on Micromechatronics and Human Science, 1999. 